\title{
Surface charge induced Dirac band splitting in a charge density wave material $\left(\mathrm{TaSe}_{4}\right)_{2} \mathrm{I}$
}

\author{
Hemian Yi, ${ }^{1}$ Zengle Huang $\odot,{ }^{2}$ Wujun Shi, ${ }^{3}$ Lujin Min $\odot,{ }^{1}$ Rui Wu ${ }^{4}$ C. M. Polley, ${ }^{5}$ Ruoxi Zhang, ${ }^{1}$ \\ Yi-Fan Zhao, ${ }^{1}$ Ling-Jie Zhou, ${ }^{1}$ J. Adell $\odot,{ }^{5}$ Xin Gui, ${ }^{6}$ Weiwei Xie, ${ }^{7}$ Moses H. W. Chan, ${ }^{1}$ Zhiqiang Mao, ${ }^{1}$ \\ Zhijun Wang $\odot{ }^{8,9}$ Weida Wu ${ }^{8},{ }^{2}$ and Cui-Zu Chang ${ }^{1}{ }^{1, *}$ \\ ${ }^{1}$ Department of Physics, The Pennsylvania State University, University Park, Pennsylvania 16802, USA \\ ${ }^{2}$ Department of Physics and Astronomy, Rutgers University, Piscataway, New Jersey 08854, USA \\ ${ }^{3}$ Center for Transformative Science and Shanghai High Repetition Rate XFEL and Extreme Light Facility (SHINE), ShanghaiTech \\ University, Shanghai 201210, China \\ ${ }^{4}$ Beijing Academy of Quantum Information Sciences, Beijing 100193, China \\ ${ }^{5}$ MAX IV Laboratory, Lund University, 22100 Lund, Sweden \\ ${ }^{6}$ Department of Chemistry, Princeton University, Princeton, New Jersey, 08540, USA \\ ${ }^{7}$ Department of Chemistry and Chemical Biology, Rutgers University, Piscataway, New Jersey 08854, USA \\ ${ }^{8}$ Beijing National Laboratory for Condensed Matter Physics and Institute of Physics, Chinese Academy of Sciences, Beijing 100190, China \\ ${ }^{9}$ University of Chinese Academy of Sciences, Beijing 100049, China
}

(Received 3 December 2020; accepted 8 March 2021; published 23 March 2021)

\begin{abstract}
$\left(\mathrm{TaSe}_{4}\right)_{2} \mathrm{I}$, a quasi-one-dimensional (1D) crystal, shows a characteristic temperature-driven metal-insulator phase transition. Above the charge density wave (CDW) temperature $T_{c},\left(\mathrm{TaSe}_{4}\right)_{2} \mathrm{I}$ has been predicted to harbor a Weyl semimetal phase. Below $T_{c}$, it becomes an axion insulator. Here, we performed angle-resolved photoemission spectroscopy measurements on the (110) surface of $\left(\mathrm{TaSe}_{4}\right)_{2} \mathrm{I}$ and observed two sets of Dirac-like energy bands in the first Brillouin zone, which agree well with our first-principles calculations. Moreover, we found that each Dirac band exhibits an energy splitting of hundreds of millielectron volts under certain circumstances. In combination with core level measurements, our theoretical analysis showed that this Dirac band splitting is a result of surface charge polarization due to the loss of surface iodine atoms. Our findings here shed light on the interplay between band topology and CDW order in Peierls compounds and will motivate more studies on topological properties of strongly correlated quasi-1D materials.
\end{abstract}

DOI: 10.1103/PhysRevResearch.3.013271

\section{INTRODUCTION}

Topological semimetals, which are characterized by linear band crossings near the Fermi level, have attracted intense interest in the condensed matter physics community in the last decade. Based on the physical origin of the band crossing, topological semimetals are categorized as Dirac semimetals, Weyl semimetals, nodal-line semimetals, and others [1,2]. Due to the spin degeneracy, the Dirac node in Dirac semimetals is composed of two massless Weyl nodes with opposite chirality, which overlap with each other at the same momentum. By breaking either time reversal or inversion symmetry, the Dirac band can split into a pair of Weyl bands. The Dirac semimetal state was discovered in $\mathrm{Na}_{3} \mathrm{Bi}$ [3] and $\mathrm{Cd}_{3} \mathrm{As}_{2}$ [4-6]. The noncentrosymmetric Weyl semimetal state was observed in TaAs [7-11], while the magnetic Weyl semimetal state was recently discovered in $\mathrm{Co}_{3} \mathrm{Sn}_{2} \mathrm{~S}_{2}[12,13]$

\footnotetext{
*Corresponding author: cxc955@psu.edu

Published by the American Physical Society under the terms of the Creative Commons Attribution 4.0 International license. Further distribution of this work must maintain attribution to the author(s) and the published article's title, journal citation, and DOI.
}

and $\mathrm{Co}_{2} \mathrm{MnGa}$ [14]. The observation of the Dirac or Weyl nodes and/or the surface Fermi arcs in angle-resolved photoemission spectroscopy (ARPES) measurements have been considered as direct evidence for these topological semimetal phases. Prior studies on topological semimetals have focused on three-dimensional (3D) crystals, which can be theoretically described by the single-particle model. More recently, the Weyl semimetal phase coupled with the charge density wave (CDW) order has been theoretically predicted [15,16] and recently claimed to be observed [17] in correlated quasi-onedimensional (1D) halogen transition metal tetrachalcogenide $\left(\mathrm{TaSe}_{4}\right)_{2} \mathrm{I}$ crystals.

$\left(\mathrm{TaSe}_{4}\right)_{2} \mathrm{I}$ is a prototype quasi-1D Peierls compound [18]. Its conventional unit cell consists of two $\mathrm{TaSe}_{4}$ chains parallel to the $c$ axis, which are separated by an iodine (I) atom plane [Fig. 1(a)]. In the $\mathrm{TaSe}_{4}$ chain, each Ta atom is sandwiched by two rectangular selenium units. The dihedral angle between adjacent rectangles is $45^{\circ}$, so this unique stacking order makes $\mathrm{TaSe}_{4}$ chains show a screwlike symmetry, and thus, $\left(\mathrm{TaSe}_{4}\right)_{2} \mathrm{I}$ is known as a chiral crystal [19]. $\left(\mathrm{TaSe}_{4}\right)_{2} \mathrm{I}$ undergoes a Peierls transition at $T \sim 263 \mathrm{~K}[17,20]$. Above the CDW temperature $T_{c}=263 \mathrm{~K}$, first-principles calculations showed $\left(\mathrm{TaSe}_{4}\right)_{2} \mathrm{I}$ to be a Weyl semimetal, originating from the chiral structure-induced inversion symmetry breaking. Below $T_{c}$, a CDW gap of hundreds of millielectron volts 

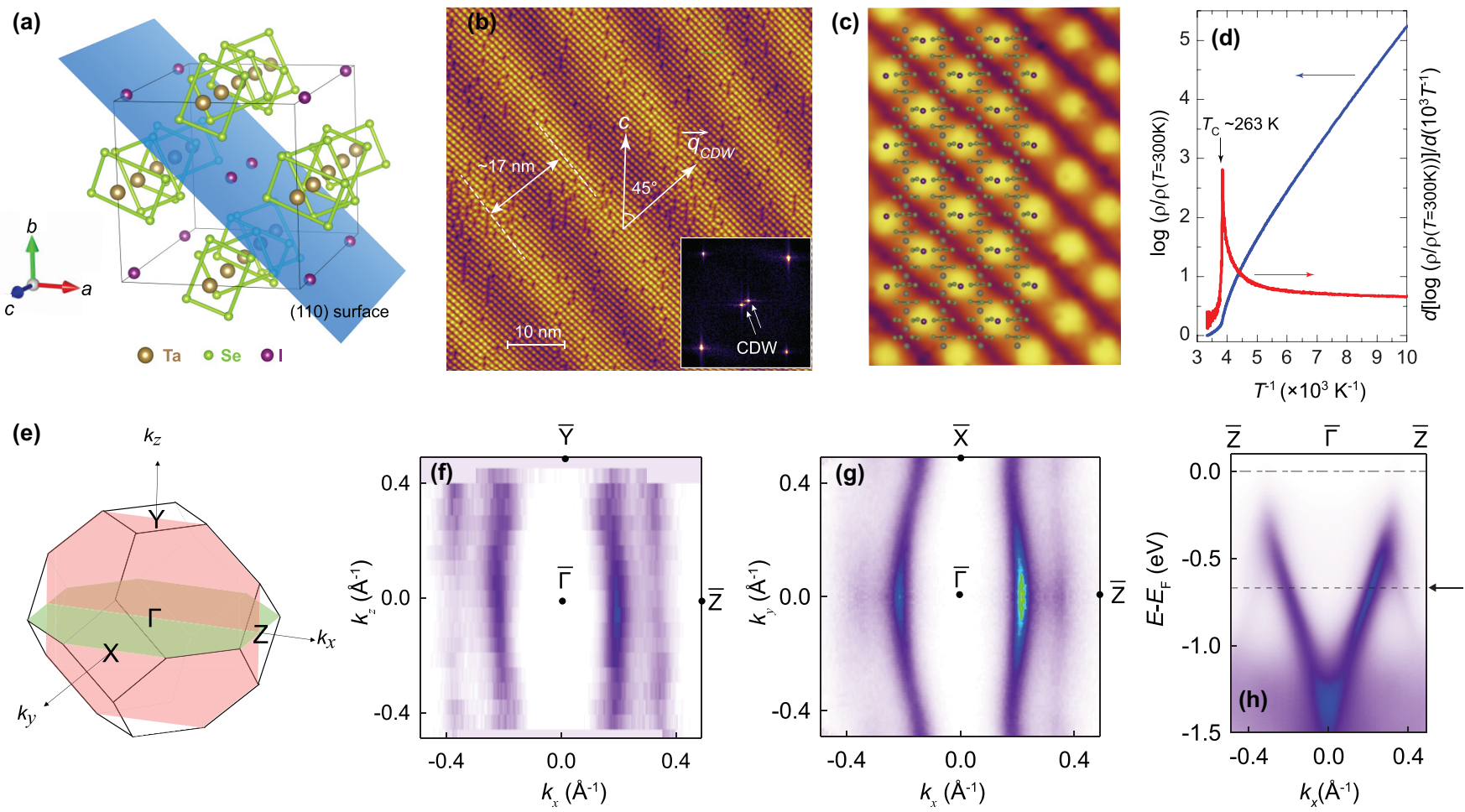

FIG. 1. Crystal structure and sample characterization. (a) Crystal structure of $\left(\mathrm{TaSe}_{4}\right)_{2} \mathrm{I}$. The (110) surface is shown in blue. (b) Scanning tunneling microscopy (STM) image of $\left(\mathrm{TaSe}_{4}\right)_{2} \mathrm{I}(110)$ surface. The $c$ axis of the crystal and the charge-density-wave (CDW) wavevector $\vec{q}_{\mathrm{CDW}}$ are shown. This image is acquired at $-1 \mathrm{~V}$ and current of $30 \mathrm{pA}$. Inset: the Fourier transform of the STM image. (c) Enlarged STM image of the green dashed rectangle area shown in (b). About 50\% of iodine atoms remain on the freshly cleaved (110) surface. (d) The logarithmic normalized longitudinal resistivity $\rho / \rho(T=300 \mathrm{~K})$ (blue) and its derivative (red) as a function of $10^{3} T^{-1}$. (e) Bulk Brillouin zone of $\left(\mathrm{TaSe}_{4}\right)_{2} \mathrm{I}$ and the surface planes on which the Fermi surface mappings are taken. (f) Constant energy contour (CEC) mapping acquired on the $\overline{\mathrm{Y} \Gamma \mathrm{Z}}$ plane at $E-E_{F}=-0.6 \mathrm{eV}$. (g) CEC mapping acquired on the $\overline{\mathrm{X} \Gamma \mathrm{Z}}$ plane at $E-E_{F}=-0.6 \mathrm{eV}$. (h) Angle-resolved photoemission spectroscopy (ARPES) band map of $\left(\mathrm{TaSe}_{4}\right)_{2} \mathrm{I}$ measured along the $\overline{\Gamma Z}$ direction. The linear horizontal (LH) polarized light with photon energy of $23 \mathrm{eV}$ is used in $(\mathrm{g})$ and $(\mathrm{h})$.

opens near the Fermi level, which can drive $\left(\mathrm{TaSe}_{4}\right)_{2} \mathrm{I}$ into an axion insulator state [15-17]. Unlike the axion insulator state realized in molecular beam epitaxy grown magnetic topological insulator sandwich heterostructures, where the gap opening at the Dirac point is induced by internal magnetization [21-23], the axion insulator realized here arises from the formation of the CDW order in a Weyl semimetal [24-26]. One recent transport study on $\left(\mathrm{TaSe}_{4}\right)_{2} \mathrm{I}$ claimed that the axion insulator state indeed appears at low temperatures (LTs) [17]. Prior ARPES studies on $\left(\mathrm{TaSe}_{4}\right)_{2} \mathrm{I}$ have interpreted the gap near the Fermi level as a polaron gap, while the CDW gap opens away from the Fermi level [27,28]. The reversed V-shaped band structure has been observed in the $\left(\mathrm{TaSe}_{4}\right)_{2} \mathrm{I}$ (110) surface along the $\overline{\Gamma Z}$ direction [Fig. 1(b)], which has been claimed to be induced by competing periodic potentials (i.e., the interaction between the two adjacent $\mathrm{TaSe}_{4}$ chains in one conventional unit cell) [29]. To date, the linearly dispersed energy bands and their electronic nodes have not been resolved in $\left(\mathrm{TaSe}_{4}\right)_{2} \mathrm{I}$.

In this paper, we report the observation of two sets of Dirac-like energy bands along the $\overline{\Gamma Z}$ direction, revealed through synchrotron ARPES measurements on the $\left(\mathrm{TaSe}_{4}\right)_{2} \mathrm{I}$ (110) surface. This result agrees well with our first-principles calculations. We further found that the Dirac band exhibits an energy splitting of hundreds of millielectron volts under certain circumstances. The circular dichroism (CD) effect of the Dirac band splitting is also studied, which further validates the observed Dirac band splitting to be an intrinsic property of $\left(\mathrm{TaSe}_{4}\right)_{2} \mathrm{I}$. We show that the band splitting observed here is not the spin splitting of energy bands due to spin-orbit coupling (SOC), which is beyond the resolution of our ARPES. By combining the core level measurements and theoretical calculations, we demonstrate that the Dirac band splitting is a result of surface charge polarization due to the loss of iodine atoms. The observation of the electronic nodes and the Dirac band splitting will help us comprehensively understand the correlated topological properties in this CDW semimetal $\left(\mathrm{TaSe}_{4}\right)_{2} \mathrm{I}$.

\section{SAMPLE CHARACTERIZATIONS AND EXPERIMENTAL DETAILS}

The quasi-1D $\left(\mathrm{TaSe}_{4}\right)_{2} \mathrm{I}$ single crystals used in this paper were synthesized using the chemical transport method [30], with a typical size of $0.8 \times 0.8 \times 5 \mathrm{~mm}^{3}$. The $\left(\mathrm{TaSe}_{4}\right)_{2} \mathrm{I}$ single crystals were characterized by the $(n n 0)$ scattering peaks from x-ray diffraction (XRD) measurements (see Fig. S2(c) in the Supplemental Material [31]). We have also performed structural refinement based on single-crystal XRD measurement. Our results validate the chiral structure of $\left(\mathrm{TaSe}_{4}\right)_{2} \mathrm{I}$ 
(see Fig. S1 and Tables S1-S3 in the Supplemental Material [31]). The fresh (110) surface was unveiled by cleaving the $\left(\mathrm{TaSe}_{4}\right)_{2} \mathrm{I}$ crystal in the ultrahigh vacuum chamber with a base vacuum better than $5 \times 10^{-11}$ mbar. The ARPES measurements were performed at the Bloch beamline of MAX IV (Sweden) and beamline 5-2 of SSRL (USA). The CDARPES measurements were performed at the SIS beamline of SLS (Switzerland). The hemispherical Scienta DA30L analyzer was used in all our ARPES measurements. The energy and angle resolution were set to $\sim 10 \mathrm{meV}$ and $\sim 0.1^{\circ}$, respectively. The Fermi level was determined by measuring a polycrystalline gold sample. The scanning tunneling microscopy (STM) experiments were carried out in an Omicron LT-STM system. The samples were cleaved in situ in ultrahigh vacuum $\left(\sim 10^{-11} \mathrm{mbar}\right)$ and then immediately transferred into the STM head for measurements. Etched tungsten tips were treated and characterized on $\mathrm{Cu}(111)$ surface. Our first-principles calculations were performed based on density functional theory using the full-potential local-orbital basis within the generalized gradient approximation. The SOC effect is included in all our calculations.

\section{RESULTS}

We first used the LT-STM to characterize the collective CDW phase in $\left(\mathrm{TaSe}_{4}\right)_{2} \mathrm{I}$. Figures 1(b) and 1(c) show the atomically resolved STM images acquired on the freshly cleaved $\left(\mathrm{TaSe}_{4}\right)_{2} \mathrm{I}(110)$ surface at about $150 \mathrm{~K}$ (below $T_{c} \sim$ $263 \mathrm{~K}$ ). The atomic features are brighter, more clearly visualized at negative bias. This indicates the cleaved surface is the iodine (I) atom layer, in which $\sim 50 \%$ iodine atoms remain [Fig. 1(c)]. A few iodine vacancies are randomly distributed on the (110) surface. The Fourier transform of the STM image [Fig. 1(b) inset] shows two sharp superlattice spots of the CDW modulations. Long-range CDW stripes with periodicity $\sim 17 \pm 1 \mathrm{~nm}$ at $150 \mathrm{~K}$ are observed on the entire scanned area [Fig. 1(b)], which is longer than that $(\sim 10.6 \mathrm{~nm})$ from prior XRD and neutron scattering measurements [32-37]. This difference might come from the change of nesting condition because of different carrier concentrations. The orientation of the CDW modulation is $\sim 45^{\circ} \pm 0.5^{\circ}$ off the $c$ axis of the crystal (along the $\mathrm{TaSe}_{4}$ chains), indicating the CDW wavevector $\vec{q}_{\mathrm{CDW}}=(-0.027,0.027,0.054)$ is in the (110) plane. The $\vec{q}_{\mathrm{CDW}}$ value is like that in prior studies [32-37]. The stripe modulation is absent at room temperature (above $T_{c}$ ), further confirming the occurrence of the CDW transition in $\left(\mathrm{TaSe}_{4}\right)_{2} \mathrm{I}$ [31]. The CDW phase transition of $\left(\mathrm{TaSe}_{4}\right)_{2} \mathrm{I}$ is also revealed in our four-terminal electrical transport measurements. Figure 1(d) shows the logarithmic normalized longitudinal resistivity $\rho / \rho(T=300 \mathrm{~K})$ and its derivative as a function of $10^{3} T^{-1}$. A pronounced peak is observed at the CDW transition temperature $T_{c} \sim 263 \mathrm{~K}$, consistent with prior transport results on this Peierls material $[17,20]$.

Next, we showed ARPES band maps on the (110) surface of $\left(\mathrm{TaSe}_{4}\right)_{2} \mathrm{I}$ using light with photon energy from 14 to $34 \mathrm{eV}$ at room temperature. The bulk Brillouin zone of $\left(\mathrm{TaSe}_{4}\right)_{2} \mathrm{I}$ is shown in Fig. 1(e). We first focus on the $\overline{\mathrm{Y} \Gamma \mathrm{Z}}$ and $\overline{\mathrm{X} \Gamma \mathrm{Z}}$ planes. Figure 1(f) shows the constant energy contour (CEC) mapping, acquired on the $\overline{Y \Gamma Z}$ plane at $E-E_{F}=-0.6 \mathrm{eV}$, where a periodic feature is observed along the $k_{z}$ direction. The appear- ance of the oppositely bent stripe band structures confirms its $3 \mathrm{D}$ property, i.e., they originate from the bulk valence bands rather than the surface states of $\left(\mathrm{TaSe}_{4}\right)_{2} \mathrm{I}$. Figures $1(\mathrm{~g})$ and 1(h) shows the $\mathrm{CEC}$ mapping acquired on the $\overline{\mathrm{X} \Gamma \mathrm{Z}}$ plane at $E-E_{F}=-0.6 \mathrm{eV}$ and the electronic band spectra along the $\overline{\Gamma Z}$ direction measured with linear horizontal (LH) light with photon energy of $23 \mathrm{eV}$. The M-shaped band structure implies the lower branch of the Dirac band. The anisotropic electronic structures in $\left(\mathrm{TaSe}_{4}\right)_{2} \mathrm{I}$ are consistent with its quasi-1D crystal structure with two interacted neighboring $\mathrm{TaSe}_{4}$ chains in one conventional unit cell.

To further demonstrate the complete Dirac band crossings, we performed high-resolution ARPES measurements on the $\left(\mathrm{TaSe}_{4}\right)_{2} \mathrm{I}$ (110) surface using linear vertical light with the photon energy of $23 \mathrm{eV}$ at room temperature [Fig. 2(a)]. Two sets of Dirac-like energy bands are resolved, which agree well with our calculated electronic band structures [i.e., the black curves shown in Fig. 2(a)]. The ARPES band map, which was treated by normalizing the momentum distribution curves (MDCs), shows these two linearly dispersed bands with electronic nodes much clearer [Fig. 2(b)].The Dirac nodes are located at $E-E_{F}=-0.39 \mathrm{eV}$, consistent with the electron carriers derived from prior Hall measurements [17]. The two Dirac nodes are seen clearly in the MDC of the ARPES band spectra [Fig. 2(b)]. A pair of separated Weyl nodes have been predicted to appear along the $\overline{\Gamma Z}$ direction above the CDW temperature $T_{c}$ [15]. Since this splitting is on the order of a few millielectron volts, neither these two separated Weyl nodes nor the predicted Fermi surface segments on the $\left(\mathrm{TaSe}_{4}\right)_{2} \mathrm{I}$ (110) surface are resolved in our ARPES experiments. It is worth noting that the two Dirac nodes are located at $k_{D} \sim$ $\pm 0.27 \AA^{-1}$, which is equal to $\sim 1.1 \pi / c(c \sim 12.778 \AA)$. This value is slightly larger than half of the CDW wavevector $(\sim 1.055 \pi / c)$ calculated by our STM results [31], consistent with the heavily electron-doped property of $\left(\mathrm{TaSe}_{4}\right)_{2} \mathrm{I}$. Here, $\vec{q}_{\mathrm{CDW}} \sim 2.11 \pi / c$ serves as the nesting vector at the Fermi surface in the CDW phase of $\left(\mathrm{TaSe}_{4}\right)_{2} \mathrm{I}$ [28].

With the electronic nodes identified, we studied the temperature evolution of the electronic band structure along the $\overline{\Gamma Z}$ direction. The photon energy of $47 \mathrm{eV}$ was used here. We first heated the freshly cleaved $\left(\mathrm{TaSe}_{4}\right)_{2} \mathrm{I}$ sample to $T=$ $350 \mathrm{~K}$ and later cooled the samples down to $T=265$ and $120 \mathrm{~K}$, respectively. At $T=350 \mathrm{~K}$, there are no coherent band spectra observed across the Fermi level [Fig. 3(a)]. We observed a gap of $\sim 120 \mathrm{meV}$ opens at the Fermi level at $T=265 \mathrm{~K}$ [Fig. 3(b)], and it increases $(\sim 240 \mathrm{meV})$ at $T=120 \mathrm{~K}$ [Fig. 3(c)]. We speculate this gap is induced by the formation of the CDW state in the sample in addition to the presence of the polaron excitations as claimed in a prior report [27]. Based on the Peierls scenario, it is unlikely the CDW gap opens at the bulk conduction-valence band crossing points instead of the Fermi level [18]. The larger gap observed at a lower temperature can be seen from the onset of the energy distribution curve (EDC) of these ARPES spectra. A fixed peak was observed at the $\Gamma$ point, which excludes the possibility that the gap opening is induced by the band shift. The bulk valence band shows the reversed V-shaped feature at all temperatures, i.e., the intersecting point is always located at the EDC onset and moves downward with decreasing temperature. In addition, the gap opened near the Fermi level 

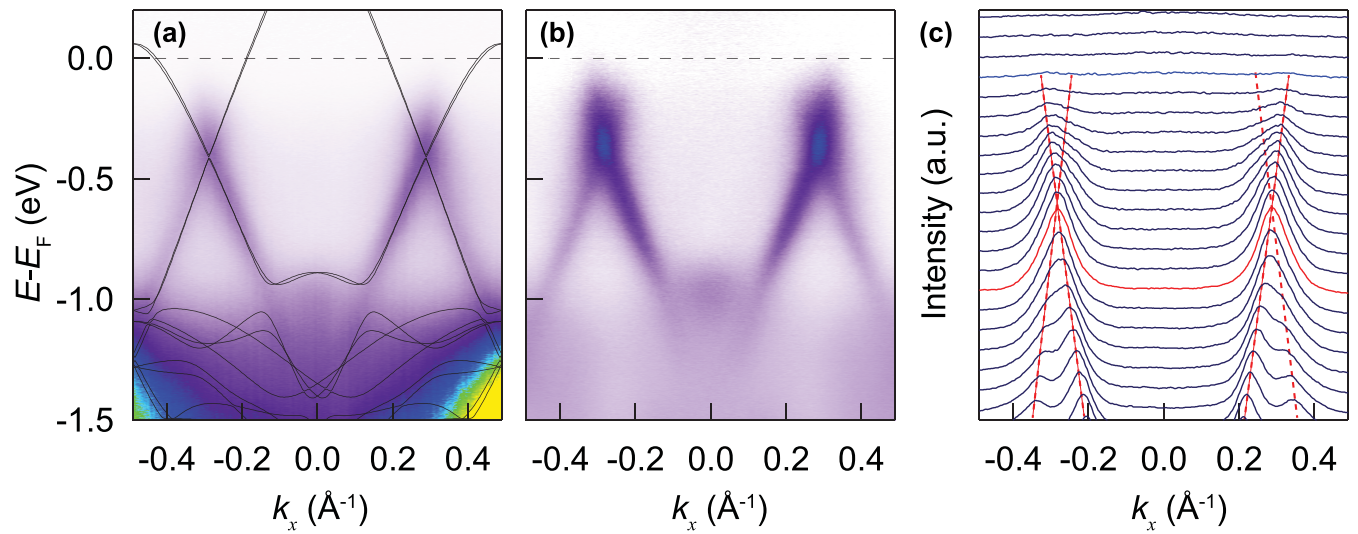

FIG. 2. Observation of Dirac bands on $\left(\mathrm{TaSe}_{4}\right)_{2} \mathrm{I}$ (110) surface. (a) Angle-resolved photoemission spectroscopy (ARPES) spectra along the $\overline{\Gamma Z}$ direction acquired at room temperature. The linear vertical light with photon energy of $23 \mathrm{eV}$ is used. The black curves are the calculated band structures. (b) The corresponding ARPES band spectra after normalizing the momentum distribution curves (MDC). (c) The MDC of the ARPES band spectra shown in (b).

appears when $T>T_{c}$. These two aspects indeed imply that the polaron gap might coexist with the CDW gap, both of which open near the Fermi level. Prior studies [27,38-40] show that the electron-phonon interaction plays a role in the formations of both CDW order and polaron quasiparticles, so we speculate the electron-phonon interaction might be responsible for the gap opened near the Fermi level. More experimental and theoretical studies are needed to clarify this issue.

In addition to the gap opened near the Fermi level, we observed a clear Dirac band splitting in these ARPES spectra acquired at $T=350,265$, and $120 \mathrm{~K}$ [Figs. 3(a) to 3(c)]. With decreasing temperature, the Dirac band splitting becomes
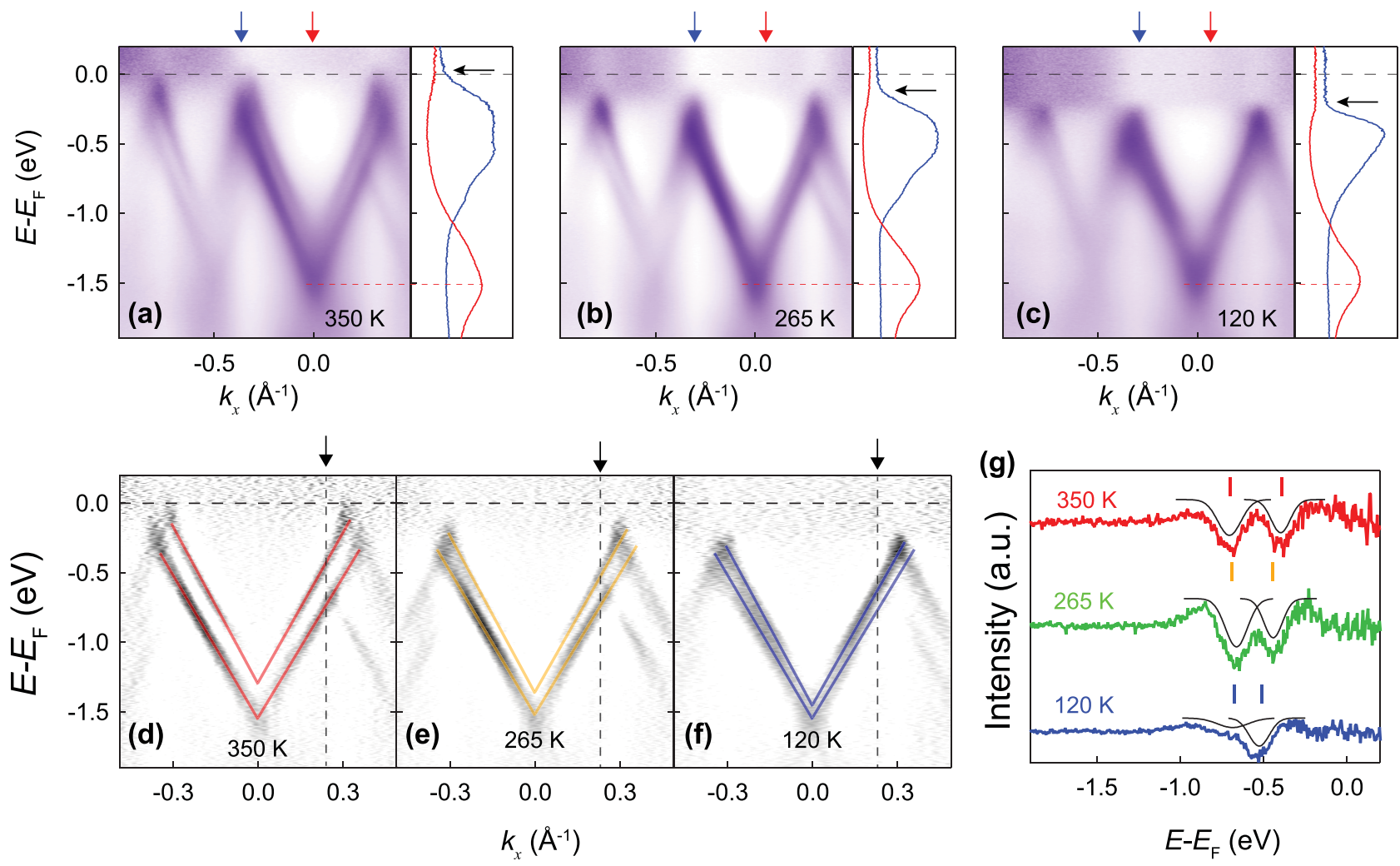

FIG. 3. Temperature dependence of Dirac band splitting on $\left(\mathrm{TaSe}_{4}\right)_{2} \mathrm{I}$ (110) surface. (a)-(c) Angle-resolved photoemission spectroscopy (ARPES) spectra along the $\overline{\Gamma Z}$ direction acquired at (a) $T=350 \mathrm{~K}$, (b) $265 \mathrm{~K}$, and (c) $120 \mathrm{~K}$. Right panels of (a)-(c): two energy distribution curve (EDC) lines extracted at two momenta marked with red and blue arrows above the left ARPES spectra. (d) and (f) The corresponding second derivative spectra of the ARPES data shown in (a)-(c). (g) The EDC line extracted at the same $k \sim 0.246 \AA^{-1}$ (i.e., $\pi / c$ ). Two dip features of each EDC line are fitted by two Gaussian functions, which can quantitatively estimate the Dirac band splitting observed on the $\left(\mathrm{TaSe}_{4}\right)_{2} \mathrm{I}(110)$ surface. 

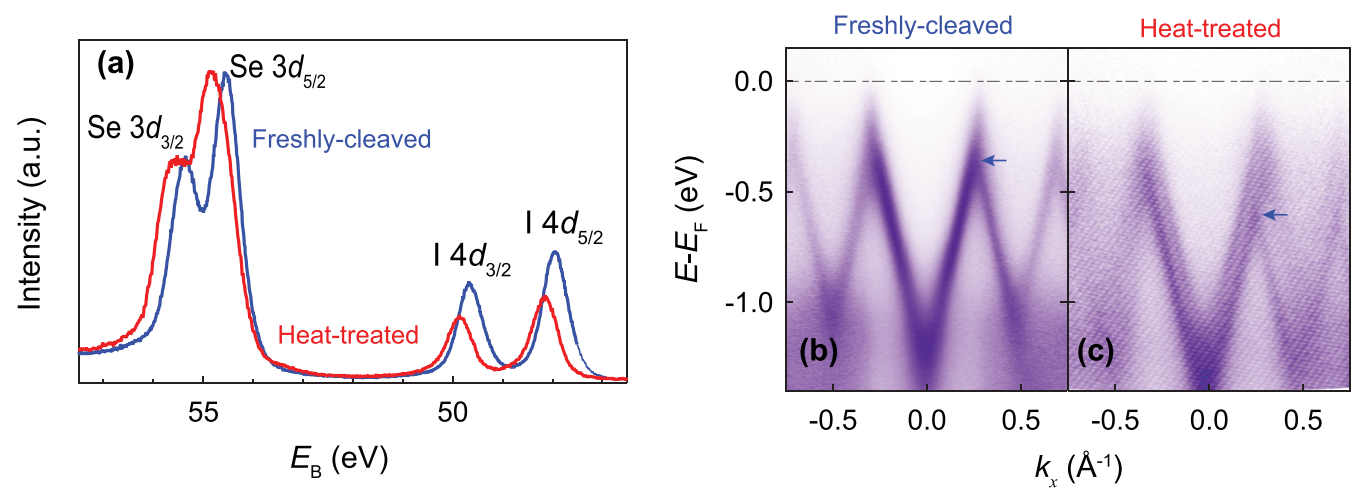

FIG. 4. Core levels of Se and iodine (I) and corresponding angle-resolved photoemission spectroscopy (ARPES) band maps of the $\left(\mathrm{TaSe}_{4}\right)_{2} \mathrm{I}$ (110) surface. (a) Core levels of Se $3 d_{3 / 2}$, Se $3 d_{5 / 2}, \mathrm{I} 4 d_{3 / 2}$, and I $4 d_{5 / 2}$. The core level photoemission measurement is performed at room temperature, and both spectra are normalized to the intensity maximum of the $\mathrm{Se} 3 d_{5 / 2}$ peak. The photon energy of $110 \mathrm{eV}$ is used. The sample is heated to $T=350 \mathrm{~K}$ for $\sim 1 \mathrm{~h}$. (b) and (c) ARPES band spectra along the $\overline{\Gamma Z}$ direction on (b) the freshly cleaved and (c) heat-treated $\left(\mathrm{TaSe}_{4}\right)_{2} \mathrm{I}(110)$ surface. The linear horizontal $(\mathrm{LH})$ polarized light with photon energy of $47 \mathrm{eV}$ is used. The blue arrows indicate the Dirac band crossing points. The ARPES measurements are performed at room temperature.

smaller. Figures 3(d) to 3(f) display the second derivative spectra of the ARPES bands, in which the Dirac band splitting are much more clearly resolved. To quantitatively study the band splitting as a function of temperature, we extracted EDCs at the same momentum $k \sim 0.246 \AA^{-1}$ (i.e., $\pi / c$ ). The two dip curves of each EDC are fitted by two Gaussian functions [Fig. 3(g)]. The energy offsets between the two split bands are $0.31,0.22$, and $0.16 \mathrm{eV}$, respectively. The theoretically proposed Weyl band splitting on the $\left(\mathrm{TaSe}_{4}\right)_{2} \mathrm{I}$ (110) surface is on the order of a few millielectron volts, which is much smaller than the linearly dispersed band splitting observed here [15]. Moreover, the Weyl band splitting is usually independent of temperature. Therefore, we concluded the Dirac band splitting observed in our experiment is not the expected Weyl band slitting on the $\left(\mathrm{TaSe}_{4}\right)_{2} \mathrm{I}$ (110) surface [15]. Since no Dirac band splitting has been observed in the room temperature ARPES band maps shown in Fig. 2 and the sample studied here has been heated to $T=350 \mathrm{~K}$, we speculated the loss of iodine atoms on the $\left(\mathrm{TaSe}_{4}\right)_{2} \mathrm{I}$ (110) surface is responsible for the Dirac band splitting.

To demonstrate the Dirac band splitting observed on the $\left(\mathrm{TaSe}_{4}\right)_{2} \mathrm{I}(110)$ surface because of the surface charge polarization due to the loss of iodine atoms, we performed core level measurements before and after the heat-treatment process, which can characterize the loss of iodine atoms and the corresponding ARPES measurements on this sample. We first performed the core level and ARPES measurements on the freshly cleaved $\left(\mathrm{TaSe}_{4}\right)_{2} \mathrm{I}(110)$ surface. For comparison, after the above measurement cycle, we heated the sample at $350 \mathrm{~K}$ for $\sim 1 \mathrm{~h}$ to artificially create more iodine vacancies on the $\left(\mathrm{TaSe}_{4}\right)_{2} \mathrm{I}(110)$ surface. When the sample was cooled down to room temperature, we performed the core level and ARPES measurements on the heat-treated sample again.

Figure 4(a) shows the core levels of Se and iodine (I) of the freshly cleaved and heat-treated $\left(\mathrm{TaSe}_{4}\right)_{2} \mathrm{I}(110)$ surfaces. Both core level spectra were normalized to the intensity maximum of the Se $3 d_{5 / 2}$ peak. Because the Se atoms are bonded to the tantalum atoms in the $\mathrm{TaSe}_{4}$ chains, the $\mathrm{Se}$ atoms are more tightly bound than the iodine atoms during the heat-treating process. We made this statement based on the below two aspects: (i) the stoichiometric ratio of the $\left(\mathrm{TaSe}_{4}\right)_{2} \mathrm{I}$ crystal is Ta $:$ Se $:$ I $=2: 8: 0.92$ (see Table S1 in Supplemental Material [31]), implying the intrinsic existence and liable formation of the iodine vacancies; (ii) the relative magnitudes of the $\mathrm{Se}$ $3 d_{3 / 2}$ and Se $3 d_{5 / 2}$ peaks do not change during the heating process, also suggesting Se is more stable. As for the I $4 d_{3 / 2}$ and I $4 d_{5 / 2}$ core levels, the peak positions, and the integrated peak area show the pronounced difference before and after the heat treatment. The reduced peak area suggests the iodine atoms indeed leave the $\left(\mathrm{TaSe}_{4}\right)_{2} \mathrm{I}$ surface. The peak positions of I $4 d_{3 / 2}$ and I $4 d_{5 / 2}$ also move to the higher binding energy, confirming the introduction of the electron carriers due to the appearance of more iodine vacancies. Figure 4(b) shows the ARPES band maps along the $\overline{\Gamma Z}$ direction on the freshly cleaved $\left(\mathrm{TaSe}_{4}\right)_{2} \mathrm{I}$ (110) surface. We observed two sets of Dirac bands in the first Brillouin zone, consistent with the band structure shown in Fig. 2. In contrast, for the heat-treated $\left(\mathrm{TaSe}_{4}\right)_{2} \mathrm{I}$ sample, the Dirac band splitting appears, and the Dirac crossing points show a downward band shift [Fig. 4(c)].

To further exclude the possibility that the Dirac band splitting observed here is from band spectra of two different $\left(\mathrm{TaSe}_{4}\right)_{2} \mathrm{I}$ pieces, we performed CD-ARPES measurements [41-45]. The CD effect in ARPES has been widely used to examine the texture of spin and/or orbital angular momentums in the Dirac surface states of topological materials [41-45]. The experimental photoemission geometry is shown in Fig. 5(a), where the incident plane is perpendicular to the $\mathrm{TaSe}_{4}$ chains, and the photoelectrons are measured with different emission angles along the $\mathrm{TaSe}_{4}$ chains (i.e., in parallel with the slit of the DA30L analyzer). Note that, because of the chiral structure, no mirror plane exists on the $\left(\mathrm{TaSe}_{4}\right)_{2} \mathrm{I}(110)$ surface. Figures 5(d) and 5(e) show the ARPES band mapping along the $\overline{\Gamma Z}$ direction obtained using the right circularly polarized (RCP) and left circularly polarized (LCP) lights, respectively. The photoemission intensity distributions of these two spectra show a clear dependence of the circular polarization of the incident photon. For instance, we observed four electronic nodes in the second Brillouin zone in Fig. 5(e), 
(a)
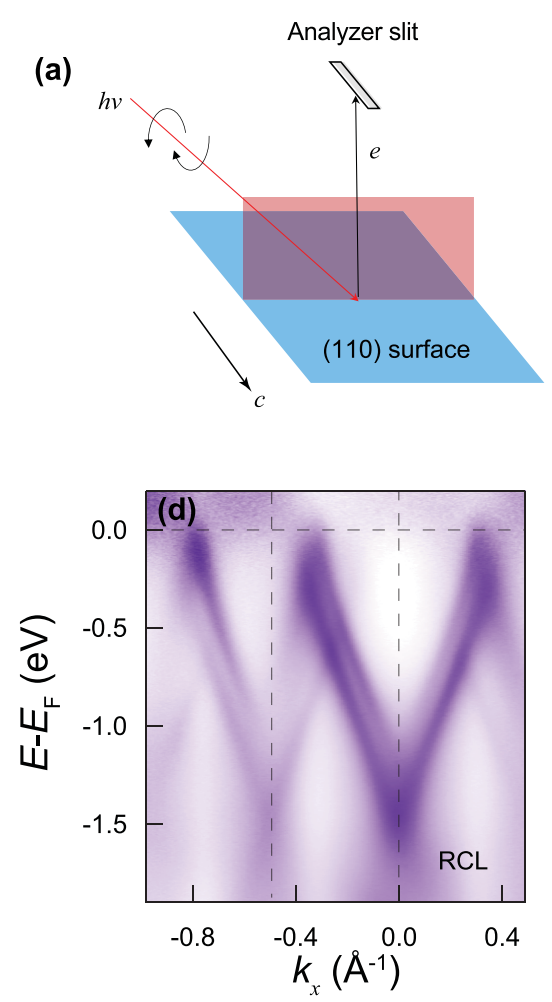
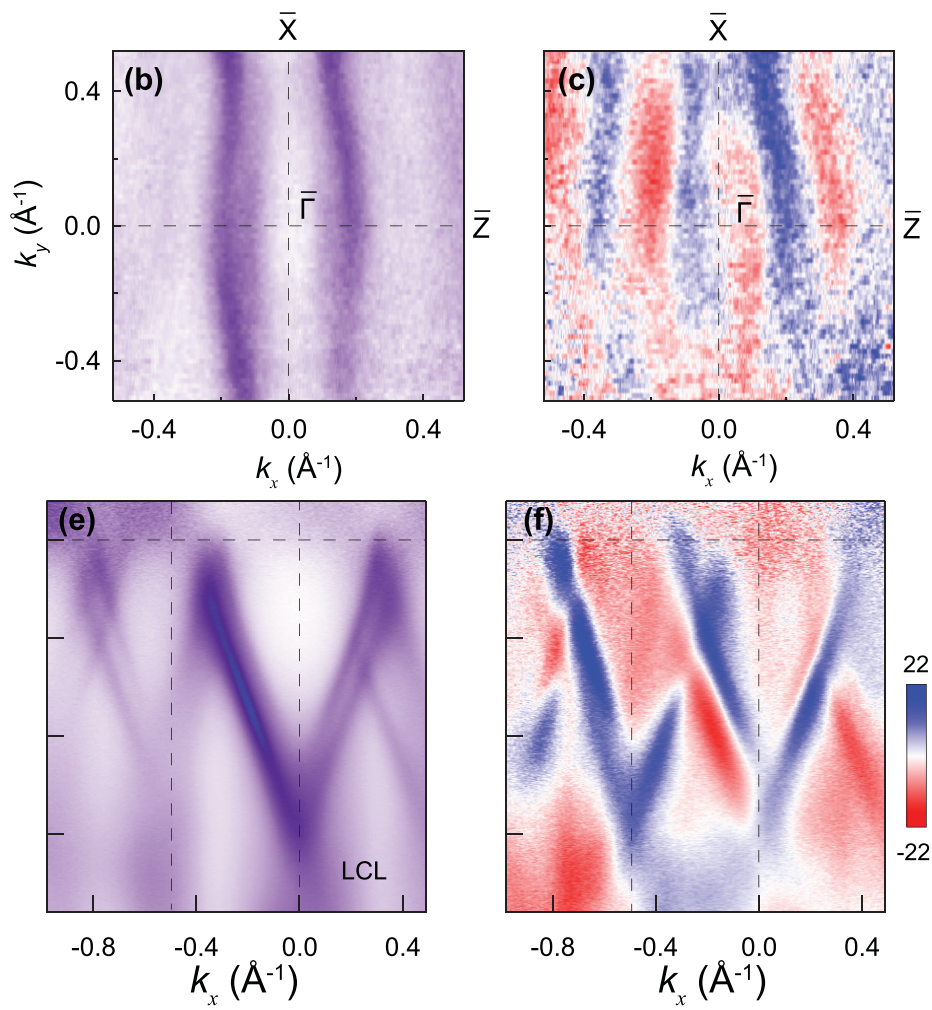

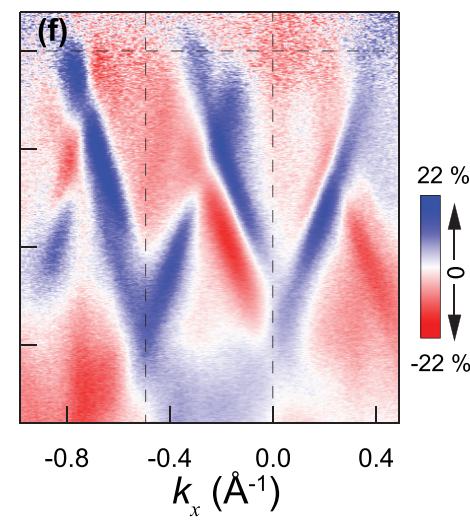

FIG. 5. Circular dichroism (CD) effect of the Dirac band splitting in $\left(\mathrm{TaSe}_{4}\right)_{2} \mathrm{I}$. (a) Experimental geometry for CD-angle-resolved photoemission spectroscopy (CD-ARPES) measurement on $\left(\mathrm{TaSe}_{4}\right)_{2} \mathrm{I}$. (b) Constant energy contour (CEC) mapping acquired on the $\overline{\mathrm{X}} \overline{\mathrm{ZZ}}$ plane at $E-E_{F}=-1.06 \mathrm{eV}$. (c) CEC mapping of the normalized CD data at $E-E_{F}=-1.06 \mathrm{eV}$. (d) and (e) ARPES spectra along the $\overline{\Gamma Z}$ direction measured with right circularly polarized (RCP) and left circularly polarized (LCP) light with photon energy of $47 \mathrm{eV}$, respectively. (f) Normalized CD spectra along the $\overline{\Gamma Z}$ direction. All ARPES data shown in (b)-(f) are taken at $T=350 \mathrm{~K}$.

some of which are absent in Fig. 5(d). To better resolve the $\mathrm{CD}$ effect, we calculated the magnitude of normalized $\mathrm{CD}$ intensity distribution using the following equation: $\mathrm{CD}=$ $\left(I_{\mathrm{RCP}}-I_{\mathrm{LCP}}\right) /\left(I_{\mathrm{RCP}}+I_{\mathrm{LCP}}\right)$, where $I_{\mathrm{RCP}}\left(I_{\mathrm{LCP}}\right)$ is the intensity of photoemission measured using the RCP (LCP) light. The observation of the alternating red-blue intensity distribution further proves the existence of the $\mathrm{CD}$ effect in the electronic structure of $\left(\mathrm{TaSe}_{4}\right)_{2} \mathrm{I}$ [Fig. 5(f)]. The CD effect can also be seen from the CEC mapping acquired on the $\overline{\mathrm{X} \Gamma \mathrm{Z}}$ plane at $E-E_{F}=-1.06 \mathrm{eV}$ [Figs. 5(b) and 5(c)]. These results suggest (i) the CD intensity of two Dirac bands located at $\pm 0.27 \AA^{-1}$ shows the left-right antisymmetry, and (ii) the sign of the $\mathrm{CD}$ intensity distribution is also reversed for these two sets of Dirac bands. Therefore, the observed Dirac band splitting is an intrinsic property of heat-treated $\left(\mathrm{TaSe}_{4}\right)_{2} \mathrm{I}$ and arises from the iodine atom loss of heat treatment.

\section{DISCUSSION}

To understand the origin of the Dirac band splitting observed in $\left(\mathrm{TaSe}_{4}\right)_{2} \mathrm{I}$, we performed first-principles calculations on $\left(\mathrm{TaSe}_{4}\right)_{2} \mathrm{I}$ on (110)-terminated slab structures with different amounts of iodine atoms on (110) surface. Since the cleaving process occurs at the iodine termination, the iodine atoms can easily leave from the cleaved surface after heating [Fig. 6(a)]. Figures 6(b) to 6(d) show the calculated electronic band structures with 100,50 , and $0 \%$ residual iodine atoms on the $\left(\mathrm{TaSe}_{4}\right)_{2} \mathrm{I}$ (110) surface, respectively. The surface spectra are obtained by projecting the energy bands of the slab calculation onto different surface terminations. In Figs. 6(b) to 6(d), the size of the red (blue) dots represents the fraction of electronic charge on the outermost (second outermost) $\mathrm{TaSe}_{4}$ chains, while the size of the gray dots represents the electronic charge on the rest bulk atoms.

For an ideal cleaving of the crystal plane, $50 \%$ of iodine atoms may stay on the (110) surface (i.e., charge neutrality) [Fig. 1(c)], and the Dirac bands coincide with the bulk bands, and no band splitting appears [Fig. 6(c)]. For a cleaved (110) surface with $100 \%$ iodine atoms and without iodine atoms (i.e., surface charge polarization), very significant upward and downward shifts of surface Dirac bands are seen [Figs. 6(b) and $6(\mathrm{~d})]$. We, therefore, attributed the Dirac band splitting observed in our ARPES measurements (Fig. 3-5) to the formation of surface charge due to the loss of iodine atoms in the surface layers. While $50 \%$ iodine surface maintains electrical neutrality, more or fewer iodine atoms at the surface would introduce a static electronic field, which is perpendicular to the surface. The static electric field could separate the surface Dirac states from the bulk states. More (fewer) adsorbed iodine atoms would drive the outermost Dirac bands to higher (lower) energy. We noted that the opposite $\mathrm{CD}$ effect observed in the outermost and second outermost $\left(\mathrm{TaSe}_{4}\right)_{2} \mathrm{I}$ layers [Figs. 5(c) and 5(f)] might be a result of the $c / 2$ shift between these two layers and/or the appearance of 


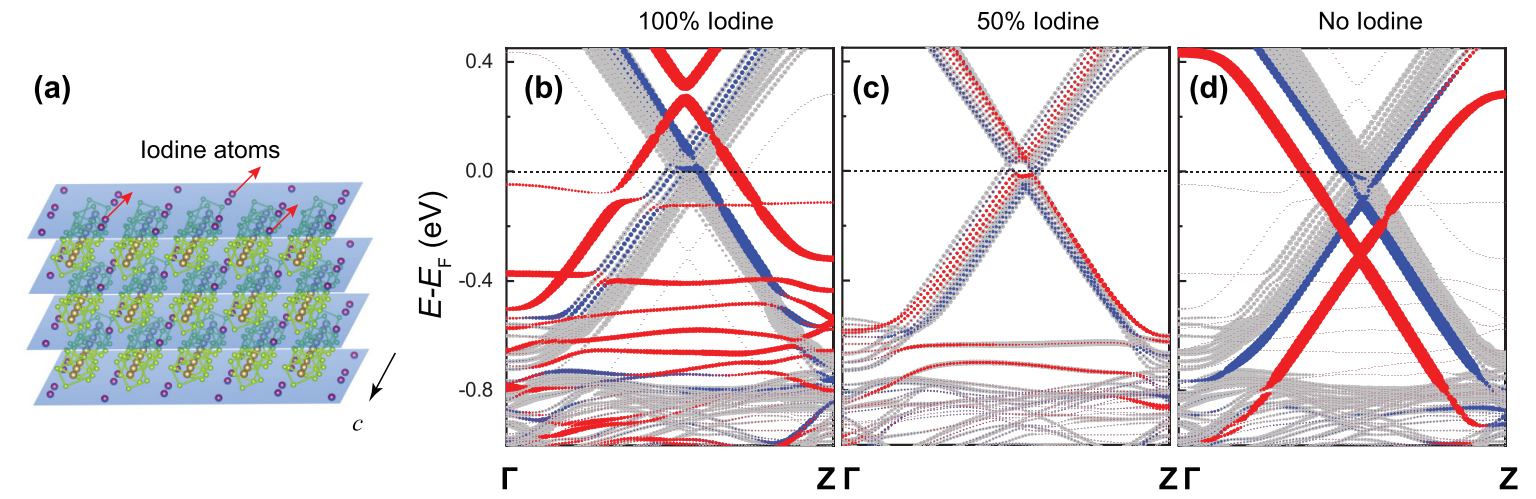

FIG. 6. Surface charge-induced Dirac band splitting on the $\left(\mathrm{TaSe}_{4}\right)_{2} \mathrm{I}(110)$ surface. (a) Shechamic for the volatile iodine atoms on the $\left(\mathrm{TaSe}_{4}\right)_{2} \mathrm{I}$ (110) surface. (b)-(d) Calculated band structures of $\left(\mathrm{TaSe}_{4}\right)_{2} \mathrm{I}$ along the $\Gamma \mathrm{Z}$ direction with the integrated $k_{z}$. (a) $100 \%$, (b) $50 \%$, and (c) $0 \%$ of iodine atoms on the $\left(\mathrm{TaSe}_{4}\right)_{2} \mathrm{I}(110)$ surface. The size of the red (blue) dots represents the fraction of electronic charge on the outermost (second outermost) $\mathrm{TaSe}_{4}$ layers, while the size of the gray dots represents that of the rest bulk atoms.

the static electric field-induced Rashba-type band splitting in the $\left(\mathrm{TaSe}_{4}\right)_{2} \mathrm{I}$ surface layers. Finally, this also explains why the magnitude of the Dirac band splitting becomes smaller with decreasing temperature [Figs. 3(d)-3(g)]: Our transport results show that the sample becomes more insulating at lower temperatures [Fig. 1(d)]; this will weaken the screening effect and reduce the electrical potential gradient between the outermost and second outermost layers and lead to a smaller Dirac band splitting.

\section{CONCLUSIONS}

In summary, we observed the Dirac-like bands in the first Brillouin zone of $\left(\mathrm{TaSe}_{4}\right)_{2} \mathrm{I}$ (110) surface, consistent with our first-principles calculations. By heat-treating the freshly cleaved $\left(\mathrm{TaSe}_{4}\right)_{2} \mathrm{I}$ (110) surface, we found Dirac band splitting, which based on our core level measurements and theoretical analysis is attributed to the loss of iodine atoms on the surface. The observed Dirac band splitting show a strong circular dichroism effect, validating that the Dirac band splitting observed in $\left(\mathrm{TaSe}_{4}\right)_{2} \mathrm{I}$ is an intrinsic property. Our findings shed light on the interaction between the Dirac bands and CDW order in Peierls compounds and provide insights in the field of strongly correlated topological materials.

\section{ACKNOWLEDGMENTS}

The authors would like to thank C. X. Liu, Z. Wang, B. H. Yan, D. Xiao, Y. T. Cui, J. Yan, and X. D. Xu for helpful discussion. This paper is primarily supported by the NSF-CAREER Award (Grant No. DMR-1847811), including the ARPES measurements, electrical transport measurements, and data analysis. The sample characterization is partially supported by a DOE Grant (Grant No. DE-SC0019064). C.-Z.C. also acknowledges the support from the Gordon and Betty Moore Foundation's EPiQS Initiative (Grant No. GBMF9063 to C.-Z.C.) and a Penn State NSF-MRSEC Grant (Grant No. DMR-2011839). L.J.M. and Z.Q.M. acknowledge the support by a Penn State NSF-MRSEC Grant (Grant No. DMR2011839). W.W. and Z.H. acknowledge the support from the ARO Award (Grant No. W911NF-20-1-0108). Use of the Stanford Synchrotron Radiation Lightsource, SLAC National Accelerator Laboratory, is supported by the US Department of Energy, Office of Science, Office of Basic Energy Sciences under Contract No. DE-AC02-76SF00515. We also acknowledge the Paul Scherrer Institut (PSI), Villigen, Switzerland, for provision of synchrotron radiation beamtime at beamline SIS of the SLS and would like to thank Plumb Nicholas Clark and Junzhang Ma for assistance.
[1] N. P. Armitage, E. J. Mele, and A. Vishwanath, Weyl and Dirac semimetals in three-dimensional solids, Rev. Mod. Phys. 90, 015001 (2018).

[2] H. M. Weng, X. Dai, and Z. Fang, Topological semimetals predicted from first-principles calculations, J. Phys. Condens. Matter 28, 303001 (2016).

[3] Z. K. Liu, B. Zhou, Y. Zhang, Z. J. Wang, H. M. Weng, D. Prabhakaran, S. K. Mo, Z. X. Shen, Z. Fang, X. Dai, Z. Hussain, and Y. L. Chen, Discovery of a three-dimensional topological Dirac semimetal, $\mathrm{Na}_{3} \mathrm{Bi}$, Science 343, 864 (2014).

[4] Z. K. Liu, J. Jiang, B. Zhou, Z. J. Wang, Y. Zhang, H. M. Weng, D. Prabhakaran, S. K. Mo, H. Peng, P. Dudin, T. Kim, M. Hoesch, Z. Fang, X. Dai, Z. X. Shen, D. L. Feng, Z. Hussain, and Y. L. Chen, A stable three-dimensional topological Dirac semimetal $\mathrm{Cd}_{3} \mathrm{As}_{2}$, Nat. Mater. 13, 677 (2014).
[5] S. Borisenko, Q. Gibson, D. Evtushinsky, V. Zabolotnyy, B. Buchner, and R. J. Cava, Experimental Realization of a ThreeDimensional Dirac Semimetal, Phys. Rev. Lett. 113, 027603 (2014).

[6] M. Neupane, S. Y. Xu, R. Sankar, N. Alidoust, G. Bian, C. Liu, I. Belopolski, T. R. Chang, H. T. Jeng, H. Lin, A. Bansil, F. Chou, and M. Z. Hasan, Observation of a three-dimensional topological Dirac semimetal phase in high-mobility $\mathrm{Cd}_{3} \mathrm{As}_{2}$, Nat. Commun. 5, 3786 (2014).

[7] S. M. Huang, S. Y. Xu, I. Belopolski, C. C. Lee, G. Q. Chang, B. K. Wang, N. Alidoust, G. Bian, M. Neupane, C. L. Zhang, S. Jia, A. Bansil, H. Lin, and M. Z. Hasan, A Weyl fermion semimetal with surface Fermi arcs in the transition metal monopnictide TaAs class, Nat. Commun. 6, 7373 (2015). 
[8] H. M. Weng, C. Fang, Z. Fang, B. A. Bernevig, and X. Dai, Weyl Semimetal Phase in Noncentrosymmetric TransitionMetal Monophosphides, Phys. Rev. X 5, 011029 (2015).

[9] L. X. Yang, Z. K. Liu, Y. Sun, H. Peng, H. F. Yang, T. Zhang, B. Zhou, Y. Zhang, Y. F. Guo, M. Rahn, D. Prabhakaran, Z. Hussain, S. K. Mo, C. Felser, B. Yan, and Y. L. Chen, Weyl semimetal phase in the non-centrosymmetric compound TaAs, Nat. Phys. 11, 728 (2015).

[10] B. Q. Lv, H. M. Weng, B. B. Fu, X. P. Wang, H. Miao, J. Ma, P. Richard, X. C. Huang, L. X. Zhao, G. F. Chen, Z. Fang, X. Dai, T. Qian, and H. Ding, Experimental Discovery of Weyl Semimetal TaAs, Phys. Rev. X 5, 031013 (2015).

[11] S. Y. Xu et al., Discovery of a Weyl fermion semimetal and topological Fermi arcs, Science 349, 613 (2015).

[12] D. F. Liu, A. J. Liang, E. K. Liu, Q. N. Xu, Y. W. Li, C. Chen, D. Pei, W. J. Shi, S. K. Mo, P. Dudin, T. Kim, C. Cacho, G. Li, Y. Sun, L. X. Yang, Z. K. Liu, S. S. P. Parkin, C. Felser, and Y. L. Chen, Magnetic Weyl semimetal phase in a Kagome crystal, Science 365, 1282 (2019).

[13] N. Morali, R. Batabyal, P. K. Nag, E. K. Liu, Q. A. Xu, Y. Sun, B. H. Yan, C. Felser, N. Avraham, and H. Beidenkopf, Fermi-arc diversity on surface terminations of the magnetic Weyl semimetal $\mathrm{Co}_{3} \mathrm{Sn}_{2} \mathrm{~S}_{2}$, Science 365, 1286 (2019).

[14] I. Belopolski et al., Discovery of topological Weyl fermion lines and drumhead surface states in a room temperature magnet, Science 365, 1278 (2019).

[15] W. Shi, B. J. Wieder, H. L. Meyerheim, Y. Sun, Y. Zhang, Y. Li, L. Shen, Y. Qi, L. Yang, J. Jena, P. Werner, K. Koepernik, S. Parkin, Y. Chen, C. Felser, B. A. Bernevig, and Z. Wang, A charge-density-wave topological semimetal, Nat. Phys. 17, 381 (2021).

[16] X.-P. Li, K. Deng, B. Fu, Y. Li, D. Ma, J. Han, J. Zhou, S. Zhou, and Y. Yao, Type-III Weyl semimetals: $\left(\mathrm{TaSe}_{4}\right)_{2} \mathrm{I}$, Phys. Rev. B 103, L081402 (2021).

[17] J. Gooth, B. Bradlyn, S. Honnali, C. Schindler, N. Kumar, J. Noky, Y. Qi, C. Shekhar, Y. Sun, Z. Wang, B. A. Bernevig, and C. Felser, Axionic charge-density wave in the Weyl semimetal $\left(\mathrm{TaSe}_{4}\right)_{2}$ I, Nature 575, 315 (2019).

[18] P. Monceau, Electronic crystals: an experimental overview, Adv. Phys. 61, 325 (2012).

[19] G. Q. Chang, B. J. Wieder, F. Schindler, D. S. Sanchez, I. Belopolski, S. M. Huang, B. Singh, D. Wu, T. R. Chang, T. Neupert, S. Y. Xu, H. Lin, and M. Z. Hasan, Topological quantum properties of chiral crystals, Nat. Mater. 17, 978 (2018).

[20] Z. Z. Wang, M. C. Saintlager, P. Monceau, M. Renard, P. Gressier, A. Meerschaut, L. Guemas, and J. Rouxel, Chargedensity wave transport in $\left(\mathrm{TaSe}_{4}\right)_{2} \mathrm{I}$, Solid State Commun. 46, 325 (1983).

[21] D. Xiao, J. Jiang, J. H. Shin, W. B. Wang, F. Wang, Y. F. Zhao, C. X. Liu, W. D. Wu, M. H. W. Chan, N. Samarth, and C. Z. Chang, Realization of the Axion Insulator State in Quantum Anomalous Hall Sandwich Heterostructures, Phys. Rev. Lett. 120, 056801 (2018).

[22] M. Mogi, M. Kawamura, A. Tsukazaki, R. Yoshimi, K. S. Takahashi, M. Kawasaki, and Y. Tokura, Tailoring tricolor structure of magnetic topological insulator for robust axion insulator, Sci. Adv. 3, eaao1669 (2017).

[23] X. Wu, D. Xiao, C. Z. Chen, J. Sun, L. Zhang, M. H. W. Chan, N. Samarth, X. C. Xie, X. Lin, and C. Z. Chang, Scaling behavior of the quantum phase transition from a quantum-
anomalous-Hall insulator to an axion insulator, Nat. Commun. 11, 4532 (2020).

[24] Z. Wang and S. C. Zhang, Chiral anomaly, charge density waves, and axion strings from Weyl semimetals, Phys. Rev. B 87, 161107(R) (2013).

[25] B. Roy and J. D. Sau, Magnetic catalysis and axionic charge density wave in Weyl semimetals, Phys. Rev. B 92, 125141 (2015).

[26] M. D. Redell, S. Mukherjee, and W. C. Lee, Resonant plasmonaxion excitations induced by charge density wave order in a Weyl semimetal, Phys. Rev. B 93, 241201(R) (2016).

[27] L. Perfetti, H. Berger, A. Reginelli, L. Degiorgi, H. Hochst, J. Voit, G. Margaritondo, and M. Grioni, Spectroscopic Indications of Polaronic Carriers in the Quasi-One-Dimensional Conductor $\left(\mathrm{TaSe}_{4}\right)_{2} \mathrm{I}$, Phys. Rev. Lett. 87, 216404 (2001).

[28] C. Tournier-Colletta, L. Moreschini, G. Autes, S. Moser, A. Crepaldi, H. Berger, A. L. Walter, K. S. Kim, A. Bostwick, P. Monceau, E. Rotenberg, O. V. Yazyev, and M. Grioni, Electronic Instability in a Zero-Gap Semiconductor: The Charge-Density-Wave in $\left(\mathrm{TaSe}_{4}\right)_{2} \mathrm{I}$, Phys. Rev. Lett. 110, 236401 (2013).

[29] J. Voit, L. Perfetti, F. Zwick, H. Berger, G. Margaritondo, G. Gruner, H. Hochst, and M. Grioni, Electronic structure of solids with competing periodic potentials, Science 290, 501 (2000).

[30] H. Fujishita, M. Sato, S. Sato, and S. Hoshino, X-ray-diffraction study of the quasi-one-dimensional conductors $\left(M \mathrm{Se}_{4}\right)_{2} \mathrm{I}(M=$ Ta and Nb), J. Phys. C: Solid State Phys. 18, 1105 (1985).

[31] See Supplemental Material at http://link.aps.org/supplemental/ 10.1103/PhysRevResearch.3.013271 for further details regarding single XRD characterization of $\left(\mathrm{TaSe}_{4}\right)_{2} \mathrm{I}$, more sample characterizations, analysis of iodine vacancies, and more ARPES results.

[32] K. B. Lee, D. Davidov, and A. J. Heeger, X-ray-diffraction study of the CDW phase in $\left(\mathrm{TaSe}_{4}\right)_{2} \mathrm{I}$-determination of the CDW modulation amplitude, Solid State Commun. 54, 673 (1985).

[33] H. Fujishita, S. M. Shapiro, M. Sato, and S. Hoshino, A neutron-scattering study of the quasi-one-dimensional conductor $\left(\mathrm{TaSe}_{4}\right)_{2}$ I, J. Phys. C: Solid State Phys. 19, 3049 (1986).

[34] H. Requardt, M. Kalning, B. Burandt, W. Press, and R. Currat, Critical $\mathrm{x}$-ray scattering at the Peierls transition in the quasione-dimensional system $\left(\mathrm{TaSe}_{4}\right)_{2} \mathrm{I}, \mathrm{J}$. Phys. Condens. Matter $\mathbf{8}$, 2327 (1996).

[35] S. van Smaalen, E. J. Lam, and J. Ludecke, Structure of the charge-density-wave in $\left(\mathrm{TaSe}_{4}\right)_{2} \mathrm{I}, \mathrm{J}$. Phys. Condens. Matter 13, 9923 (2001).

[36] H. Fujishita, M. Sato, and S. Hoshino, Incommensurate superlattice reflections in quasi-one-dimensional conductors, $\left(\mathrm{TaSe}_{4}\right)_{2} \mathrm{I}$ and $\left(\mathrm{NbSe}_{4}\right)_{2} \mathrm{I}$, Solid State Commun. 49, 313 (1984).

[37] V. Favre-Nicolin, S. Bos, J. E. Lorenzo, J. L. Hodeau, J. F. Berar, P. Monceau, R. Currat, F. Levy, and H. Berger, Structural Evidence for Ta-Tetramerization Displacements in the ChargeDensity-Wave compound $\left(\mathrm{TaSe}_{4}\right)_{2} \mathrm{I}$ from X-Ray Anomalous Diffraction, Phys. Rev. Lett. 87, 015502 (2001).

[38] T. Valla, A. V. Fedorov, P. D. Johnson, P. A. Glans, C. McGuinness, K. E. Smith, E. Y. Andrei, and H. Berger, Quasiparticle Spectra, Charge-Density Waves, Superconductivity, and Electron-Phonon Coupling in $2 \mathrm{H}-\mathrm{NbSe}_{2}$, Phys. Rev. Lett. 92, 086401 (2004). 
[39] X. T. Zhu, Y. W. Cao, J. D. Zhang, E. W. Plummer, and J. D. Guo, Classification of charge density waves based on their nature, Proc. Natl. Acad. Sci. 112, 2367 (2015).

[40] J. T. Devreese, Polarons, Encycl. Appl. Phys. 14, 383 (1996).

[41] S. Cho, J. H. Park, J. Hong, J. Jung, B. S. Kim, G. Han, W. Kyung, Y. Kim, S. K. Mo, J. D. Denlinger, J. H. Shim, J. H. Han, C. Kim, and S. R. Park, Experimental Observation of Hidden Berry Curvature in Inversion-Symmetric Bulk $2 \mathrm{H}-\mathrm{WSe}_{2}$, Phys. Rev. Lett. 121, 186401 (2018).

[42] S. R. Park, J. Han, C. Kim, Y. Y. Koh, C. Kim, H. Lee, H. J. Choi, J. H. Han, K. D. Lee, N. J. Hur, M. Arita, K. Shimada, H. Namatame, and M. Taniguchi, Chiral Orbital-Angular Momentum in the Surface States of $\mathrm{Bi}_{2} \mathrm{Se}_{3}$, Phys. Rev. Lett. 108, 046805 (2012).
[43] Y. H. Wang, D. Hsieh, D. Pilon, L. Fu, D. R. Gardner, Y. S. Lee, and N. Gedik, Observation of a Warped Helical Spin Texture in $\mathrm{Bi}_{2} \mathrm{Se}_{3}$ from Circular Dichroism Angle-Resolved Photoemission Spectroscopy, Phys. Rev. Lett. 107, 207602 (2011).

[44] M. R. Scholz, J. Sanchez-Barriga, J. Braun, D. Marchenko, A. Varykhalov, M. Lindroos, Y. J. Wang, H. Lin, A. Bansil, J. Minar, H. Ebert, A. Volykhov, L. V. Yashina, and O. Rader, Reversal of the Circular Dichroism in Angle-Resolved Photoemission from $\mathrm{Bi}_{2} \mathrm{Te}_{3}$, Phys. Rev. Lett. 110, 216801 (2013).

[45] C. Z. Xu, Y. Liu, R. Yukawa, L. X. Zhang, I. Matsuda, T. Miller, and T. C. Chiang, Photoemission Circular Dichroism and Spin Polarization of the Topological Surface States in Ultrathin $\mathrm{Bi}_{2} \mathrm{Te}_{3}$ Films, Phys. Rev. Lett. 115, 016801 (2015). 